\title{
Responses to incidental stimuli as a function of feedback contingency
}

\author{
GERALD M. MURCH ${ }^{2}$ \\ PORTLAND STATE COLLEGE
}

The effects of incidental stimuli, defined as visual stimuli not under verbal control, upon a selection task were observed as a function of feedback contingency. Ss judged letter pairs to be valid or invalid completions of preceding letter parts. During the presentation of the letter parts, the response pair or an altemative pair of letters was intermittently presented at a thrishold ralue individually determined for each $S$. One group of Si rucived a light feedbath of performance contingent upon responding to the incidental stimuli; whereas a second group received a random noncontingent feedback. The results indicated: (a) both groups responded to the incidental stimuli; (b) feedback contingency had no effect on frequency of responding to the incidental stimuli; (c) frequency of responding to the incidental stimuli remained constant over i2 trials.

The study of discrimination without awareness, subception. and subliminal perception has been plagued since its onset witl methodological problems and considerations. Eriksen (1958) and Wiener and Schiller (1960) came to the conclusion that most studies of subliminal stimulation observed the effects of partial cues providing the necessary information for a discriminatory response. These cues were not under adequate verbal control of the $S$, primarily because of the fallibility of postexperimental methods of ascertaining the $S$ 's degree of awareness of the discriminatory stimuli (Bernstein \& Eriksen, 1965).

In a further presentation Eriksen (1960) has advanced definitions of subliminal stimuli and partial cues, the latter referred to by him as incidental stimuli. Subliminal stimuli are stimuli "whose intensity is below that where a differentiated or discriminated verbal report can be obtained [p. 281]." Incidental stimuli are ones that for the $S$ are "above the threshold but which upon questioning, the $S$ is unable to state that he used these particular stimuli as cues for his responses [p. 281]."

The practical application of subliminal stinuli proves in light of these definitions quite difficult. The failure of a $S$ to identify verbally a stimulus allows such a stimulus to be classified as an incidental stimulus, but not necessarily as a subliminal stimulus. The use of subliminal stimulus presumes some accurate method of assessing the subliminality of the stimulus beyond the ability of an $S$ to report verbally its presence.

The explanation of most claimed demonstrations of subliminal perception on the basis of incidental stimuli does not solve the problem of specifying the conditions under which such incidental stimuli may affect behavior. The effects of incidental stimuli upon response patterns are, in their own right, of interest to the study of behavior.

The present study attempts firstly to ascertain the effects of incidental stimuli upon a discriminatory response and secondly, in the case of the presence of such effects, to observe the influence of a performance feedback contingent upon responding to the incidental stimuli.

Incidental stimuli are defined as novel visual stimuli, presented below the threshold which would allow a $S$ to verbally identify the stimuli upon questioning. The term novel is included in the above definition to indicate that these incidental stimuli do not modify behavior due to the S's previous experience with the stimuli. Obviously the majority of cues utilized in perception are not under verbal control. This is due. however, to a S's extensive experience with the cues rather than the subthreshold nature of the cues.

\section{METHOD}

Forty-eight students at the Psychologisches Institut in Gottingen, West Germany, served as Ss for the present experiment.

In the first part of the experiment, individual absolute thresholds for the incidental stimuli were established. Ss entered a darkened room individually and were seated in front of the tachistoscope (Scientific Prototype, three-field Model GB). After a 5 -min dark adaptation period the $S$ was instructed to look into the viewer of the tachistoscope where a $3 \times 3 \mathrm{~cm}$ square could be seen. A fixation point was located in the center of the square. An $E$ read instructions telling the $S$ to fixate the point. The $S$ was told further that a series of letters, one after another, would be presented for very short durations and that the length of presentation of each letter would slowly be increased until he was able to name correctly the letter in question.

The presentations of the letters were begun with an exposition duration period of 1 msec. The duration was increased in increments of $0.1 \mathrm{msec}$ after each 10 stimulus presentations, until the $\mathrm{S}$ correctly identified the letters. The interstimulus interval was 250 msec. $^{3}$

A total of six letters (U, M, H, S, K, P) was used, each $2 \mathrm{~cm}$ high and drawr on white stimulus cards with a black felt Gutenbourg Signierstift through a Standardgraph Stencil 202/20. The letter was presented within the $3 \times 3 \mathrm{~cm}$ square centered at the fixation point.

The threshold value for the presentation of the incidental stimuli in the main experiment was computed by establishing the mean of the shortest durations, at which the letters were correctly identified, for the last five letters for each individual S.

The main experiment consisted of a discrimination task in which the parts of two letters, with a fixation point between them, were presented for $5 \mathrm{sec}$ in the first field of the tachistoscope. At the end of the $5 \mathrm{sec}$, a completed pair of letters, constituting an accurate completion of the previously shown letter parts was presented for $2 \mathrm{sec}$. During the 5 -sec presentation of the letter parts, a further completed letter pair, the incidental stimulus, was presented every $250 \mathrm{msec}$ (i.e., 20 times) at the threshold duration determined for that individual in the pretest. The luminance of the field with the letter parts and incidental stimuli was $3.6 \mathrm{ft}-\mathrm{c}$.

The letter parts and completed letters as well as the incidental stimuli were prepared with a Standardgraph 202/20 Stencil on white stimulus cards. The letter parts and completed letters were drawn with a black Gutenbourg Signierstift, whereas the incidental letter pair was drawn with a red Gutenbourg Signierstift. The red/white contrast ratio was less than that of the black/white ratio of the threshold determination and increased the probability that the incidental stimuli would actually remain unnoticed by the Ss (see Murch, 1965).

The letter parts, incidental letter pair and response letter pair were all presented equidistant from the fixation point so that complete retinal congruency of all letters was obtained.

The task consisted in judging the response letter pair as compatible or incompatible with the preceding letter parts. Ss indicated their discrimination response by pressing onc of two respective Morse-keys.

Pressing the left key meant that he felt the letter pair figted the preceding letter parts (+ response) and pressing the right key indicated that the $S$ felt the letter pair did not fit the preceding letter parts (-- response). 
Each $S$ received a total of 32 problems. The interval between problems was $15 \mathrm{sec}$, during which an adaptation ficld with a fixation point was presented. The order of presentation was permuted across $S$ s. On 16 of the problems the interposed incidental letter pair was the same as the letter pair presented at the end of the 5-sec period, whereas on the remaining 16 problems, the incidental letter pair was different. In all cases, however, the response letter pair and the incidental letter pair actually constituted valid completions of the preceding letter parts.

Figure 1 contains the 32 problems used in this design. Column (a) contains the letter parts. Column (b) the corresponding incidental stimuli, and Column (c) the response letter pair.

After completing the pretest threshold measurements, the Ss were assigned to onc of three groups. Assignments were made on the basis of threshold measurements such that three matched groups were formed.

Group EC (Experimental Contingent) received performance fecdback via a light locited immediately below the tachistoscope viewer. The light feedback was made contingent upon responding to the incidental stimuli. That is, if the incidental letter pair and the response letter pair were the same and the $S$ indicated the response letter pair to be valid completion of the letter parts, feedback was presented (H response). Further, if the $S$ deemed the response pair to be an invalid completion of the letter parts after the presentation of the other incidental lelter pair, feedback occurred (- - response).

In the case of Group EN (Experimental Noncontingent) a noncontingent feedback was given. The light activated with a probability of .60 on each trial independent of the response behavior of the $S$.

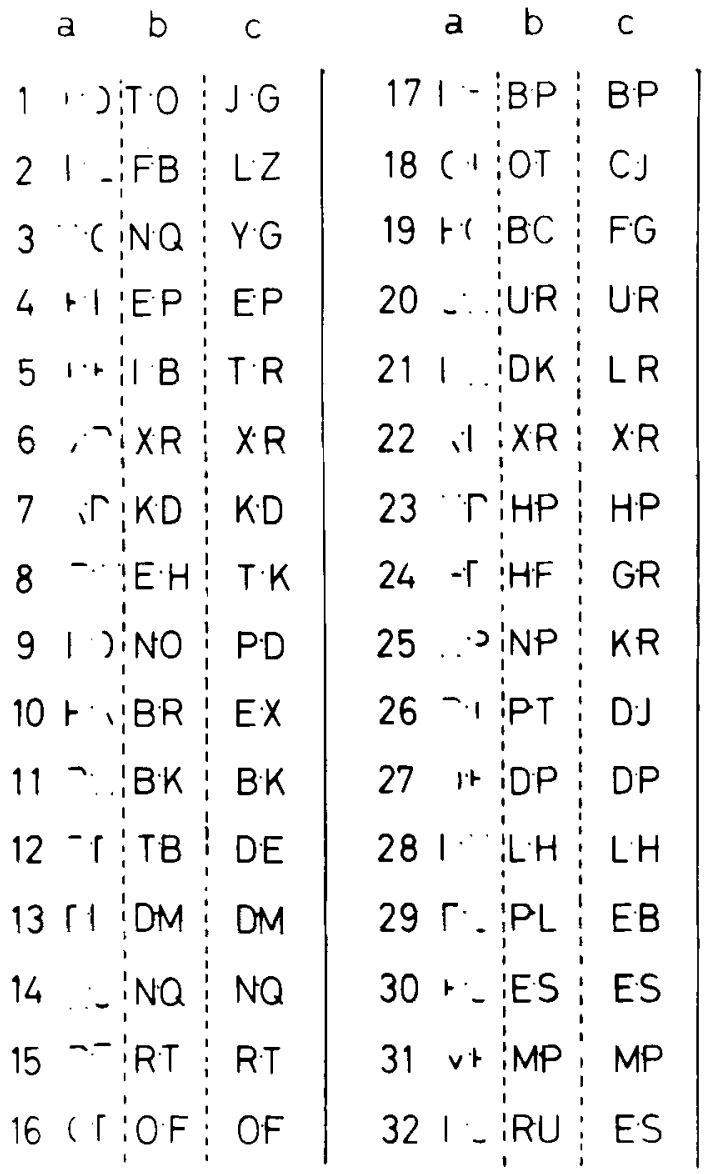

Fig. 1. Stimulus materials used in the main experiment. Column (a): Parts of letters. Column (b): Incidental stimuli. Column (c): Response letter pairs.
A third group, ( $N$ (Control Noncontingent) represented the control condition in which no incidental stimuli were presented. In this group, a blank white card was presented every 250 ntsec during the 5-sec presentation of the letter parts at the threshoid established in the pretest. Feedback occurred on a noncontingent basis with a probability of 60 on each trial.

The instructions prior to the main experiment emplasized not attempting to analyze the problem during the presentation of the letter parts. Rather, the $S$ was to fixate the point between the letter parts and respond to the response letter pair "intuitively" and as rapidly as possible. The task was represented as a study ol the reliability of first inpressions.

The program for the presentation of the stimuli and activation of the recording devices was controlled by a Massey-Iickenson Programming Unit allowing the design to function atomatically. The $\mathrm{E}$, who remaned out of sight and contact after reading the instructions, needed only to change the stimulus cards during the intertral interval.

At the end of the experiment each $S$ was asked four questions ained at establishing his ability to verbalize the incidental stimuli: (1) How did you go about choosing at response category? (2) Did you see anything during the presentation of the letter parts which made your choice casier? (3) Would you please rate on a scale between 1 and 7 the degree to which you attempted to respond intuitively? The Value 1 indicaltes complete intuitive responding and the Value 7 complete premeditated responding. (4) During the presentation of the fetter parts sometimes a letter pair was blended in for very short durations. Did you see any of these letter pairs or anything which might have been parts of these letter pairs?

If the S's answers to the above questions indicated his ability to verbalize the presence of the incidental stimuli, his data were deleted from the evaluation. This was not the cise with any of the Ss.

\section{RESULTS}

An analysis of variance was conducted with the data. The overall $F$ for the number of responses corresponding to the incidental stimuli indicates a highly significant difference between groups, $F(2,45)=21.71, p<.001$. The comparison of each experimental group with the control group ${ }^{4}$ indicales a significant difference between both $E C$ and $C N, F(2.30)=5.14 . p<.05$, and $E N$ and $C N, F(2,30)=7.04, p<.01$. In both experimental groups the presentation of the incidental stimuli had a significant effect upon response behivior.

The comparison of the two experimental conditions, EN and $E C, 1:(2,30)=.56, p>.05$, failed to yield a significant difference. This result indicates that making the light feedback contingent upon responding to the incidental stimuli had no effect upon responses to the incidental stimuli.

A possible source of this failure to observe an effect of the feedback contingency might be found in the total number of light presentations given to the contingent $S$ s (EC) as compared to $S$ s in the noncontingent group (EN) who received a light presentation on each trial with a fixed probability of .60. The comparison. however, failed to yield a significant difference in the number of light reinforcements, $\mathrm{F}(2,30)=.37, \mathrm{p}>.05$.

Reinforcement theory should predict a performance increment in the case of the group receiving contingent light feedback for responding to the incidental stimuli. To test this prediction, the 32 problems were divided into lour blocks of eight trials and a trend analysis of these data was performed. However. the analysis indicated no performance increment over trial blocks. $F(3,129)=.68, p>05$. The (iroups by Trials interaction also failed to reach a significant vilue, $F(0,129)=.67, p>.05$.

A correct response to the incidental stimuli consisted in either judging the response letter pair correct after that pair had been presented as the incidental stimulus $(++)$ or judging the response letter pair incorrect alfer the presentation of an alternative letter pair as the incidental stimulus ( $)$. It was possible that the effects of the incidental stimuli under these two conditions woukd be different. In Table I the mean number of $(t+1$ and 1 ) responses is given. Also contained in Tabk I alre the mean 
Table 1

Mean Number of Responses to the Incidental Stimuli After Presentation of the Same Letter Pzir as the Incidental Stimulus $(++)$ and After Presentation of an Alternative Letter Pair as the Incidental Stimulus (--). Mean Number of Judgments of Valid Letter Completion ( + ) and Invalid Letter Completion (-).

\section{Group}

Response

$(++)$

$(-)$

(t)

(-)

$(++) /(+)$

$(-) /(-)$

EC

EN

CN

9.0

5.6

19.5

12.4

.46 .45

numbers of $(+)$ responses. that is, the mean number of times that the response letter pair was judged correct, and the mean numbers of $($ ) responses when the letter pair was judged incorrect. The final entry in Table 1 divides the $(++)$ and $(--$, response means by the (t) and (-) means, respectively, in order to present the ratio between mean correct responses and mean numbers of judgment with that response category.

Although the means definitely favor $(++)$ rcsponses, the mean number of $(t)$ responses indicates a tendency to litilize this response more frequently. When these means are equated in order to partial out the effects of frequency, it is obvious that the larger $(++)$ means are due to the more frequent utilization of the judgment of the letter pairs to be a valid completion of the letter parts. The probabilities that a $(+)$ response would occur on a given trial were .55 for Group CN, and .52 for Group EN.

A final set of calculations involved the ratings of the degree of "intuitiveness" with which the $S$ felt he had responded to the problems. Product-moment correlations were computed between the ratings and the number of responses to the incidental stimuli. For Group EN an $r$ of -.72 ( $\mathrm{df}=14), \mathrm{p}<.005$ was obtained and for Group EC an $r$ of $-.67(\mathrm{df}=14), \mathrm{p}<.005$. The control group, $C N$, showed a correlation of $r=.25(\mathrm{df}=14), p>.05$.

As a whole these data indicate that a tendency to rate intuitively was positively related to responding to the incidental stimuli.

\section{DISCUSSION}

The mijor result of this study was that the Ss did respond to incidental visual stimuli, the presence of which were not under verbal control. Information to the $S$ on his attentiveness to the incidental stimuli in the form of a contingent light feedback, appeared to have no effect upon performance. Ss were found to respond to the incidental stimuli just as frequently without performance feedback as witl performance feedback. In most studies of discrimination learning in which the $S$ is aware of the feedback contingency or becomes aware of the feedback contingency performance improves over trials. That is to say, when the $S$ perceives a meaningful relationship between his response and the frequency of feedback, he tends to respond in a manner allowing the most frequent occurrence of feedback. In the present design Ss responded to the incidental stimuli, but did not improve over trials.

The lack of an increase in performance may be due to the relatively small number of trials (32), or due to the nature of the information conveyed to the $S$ by the incidental stimuli. If the latter possibility was the case, then it would appear that the information was not of a cognitive nature. Recent research by McNulty, Dockrill, and Levy (1967) demonstrated that the meanings of subthreshold words has no effect upon behavior. Generally speaking such observations would indicate that the incidental stimuli are not involved as central mediators of a cognitive response. Rather, they serve to predispose the visual system, perhaps at the level of the retina, to respond to or be more sensitive to certain stimuli (see Murch 1967). The effect of the stimuli on the visual system appears to require, however, a certain receptiveness on the part of the $S$. If $S s$ responded without analyzing the problems (e.g., intuitively) the incidental stimuli had a greater effect. Perhaps conscious processes are able to override the influences of the incidental stimuli. The opposite effect, that the incidental stimuli instigate conscious processes, does not, on the basis of these data, appear plausible.

\section{REFERENCES}

BERNSTEIN, I. H., \& ERIKSEN, C. W. Effects of subliminal prompting on paired-associate learning. Journal of Experimental Research in Personality, $1965,1,33-38$.

ERIKSEN, C. W. Unconscious processes. In M. Jones (Ed.), Nebraska symposium on motivation. Lincoln, Nebraska: University of Nebraska Press, 1958. Pp. 169-225.

ERIKSEN, C. W. Discrimination and learning without awareness: A methodological survey and evaluation. Psychological Review, 1960, 67, 279-300.

HABER, R. N., \& HERSHENSON, M. Effects of repeated brief exposure on the growth of a percept. Journal of Experimental Psychology, 1965, 69, 40.46.

McNULTY, J. A., DOCKRILL, F, J., \& LEVY, B. A. The subthreshold perception of stimulus meaning. American Journal of Psychology, 1967, 80,2840

MURCH, G. M. A simple laboratory demonstration of subception. British Journal of Psychology, 1965, 56, 467470.

MURCH, G. M. Temporal gradients of responses to subliminal stimuli. Psychological Record, 1967, 17, 483-492.

WEINER, M., \& SCHILLER, P. H. Subliminal perception or the perception of partial cues. Journal of Abnormal \& Social Psychology, 1960, 61, 124-137.

\section{NOTES}

1. This research was conducted at the Psychologisches Institut in Gottingen, West Germany, and was supported by a grant from the Deutsche Forschungsgesellschaft. The author wishes to express his thanks to Klaus Nippert and Josef Reinartz for their aid in conducting experiments.

2. Address: Department of Psychology, Portland State College, Portland, Oregon 97207

3. Recent research by Haber and Hershenson (1965) has indicated that the increase in the probability of recognizing and identifying a stimulus as a function of the frequency of presentation with duration held constant, reaches an asymptote at about 10 presentations.

4. A correct response was counted for $\mathrm{Ss}$ in the control group, $\mathrm{CN}$, when the response compatible with the incidental stimuli presented to the matched Ss in the experimental groups was made.

(Accepted for publication August 26, 1968.) 